\title{
Fabrication of high-sensitivity pyroelectric sensors by ion beam etching
}

\author{
$\underline{\text { Robert Rückriem }}{ }^{1}$, Reinhard Köhler ${ }^{2}$, Michael Zeuner ${ }^{1}$ \\ ${ }^{1}$ scia Systems GmbH, Annaberger Straße 240, 09125 Chemnitz \\ 2 DIAS INFRARED GMBH Pforzheimer Straße 21, 01189 Dresden
}

\section{Summary}

Ion beam etching of pyroelectric sensors made of lithium tantalate (LT) is presented in order to get a higher specific detectivity $D^{*}$ compared to standard sensors. We present the etching tool which applies argon ion beam etching in production environment. The etching homogeneity was investigated by a standard silicon oxide etch and a twelve hour silicon etch with photoresist mask. Both show a homogeneity of $+/-0.7 \%$ and a good compliance. Further investigations were done in a twelve hour process regarding the stability of the ion current regulation and ion current density. In the first two hours after process start, the main variation of power and ion current density takes place. Afterwards, the system reached a steady-state. After etching of the LT samples, an analysis of the removed material and emerged etching walls was done. Finally, the important $D^{*}$ was compared between an ion-etched and a non-ion etched pyroelectric sensor.

Keywords: Ion beam etching, pyroelectric sensors, IR sensors, production environment, lithium tantalate

\section{Introduction}

Infrared radiation sensors are often made of a pyroelectric material such as lithium niobate (LN) or lithium tantalate (LT) [1]. Based on a grown single crystal of a certain diameter, z-cut wafers of a thickness of about $300 \mu \mathrm{m}$ are sliced. A further reduction of the wafer thickness is done by a polishing process down to about $25 \mu \mathrm{m}$. Achieving a smaller thickness of the substrate is not possible due to cracks appearing due to the polishing additional removing of the wafer from a lapping substrate [2].

The specific detectivity $D^{*}$ of a piezoelectric element was found to be strongly influenced by LT thickness [3]. A further reduction of the LT thickness below $25 \mu \mathrm{m}$ in order to increase $D^{*}$ is possible by using $\mathrm{Ar}^{+}$ion beam etching, so called ion beam milling. Sensors manufactured with this technology show a two times higher $D^{*}$ required for temperature measurements or IR spectroscopy [4]. As the ion beam etching rate of $L T$ is in the range of $1 \mu \mathrm{m} / \mathrm{h}$, a longterm stable process over several hours is required to get desired thinness of the $L T$.

In this work, we present the results of the ion beam etching applied to pyroelectric sensors in order to get high $D^{*}$ values for a consumer market product. The ion beam milling tool is described and long-term stability is investigated. Furthermore, the manufactured samples were analyzed.

\section{Preparation of the lithium tantalate}

Before the ion beam etching, the LT substrates had a thickness of about $25 \mu \mathrm{m}$. The back was coated with a $\mathrm{NiCr}$ metal layer which would later act as the back electrode. The top of the LT wafer was coated with a structured photoresist (PR) mask. The substrate was mounted on a 1.5" sub-carrier which allows (1) multiple LT substrates gathering and (2) was required for mechanical support of the thin $L T$ wafers.

In Figure 1 a sketch of the integration of an ion beam etched LT sample in a pyroelectric sensor is shown. The top electrode and an optional absorption layer [5] were deposited after the ion beam etching. While the sensitive area in the center should be as thin as possible, the thicker frame is necessary for mechanical stability of the sensor chip. 


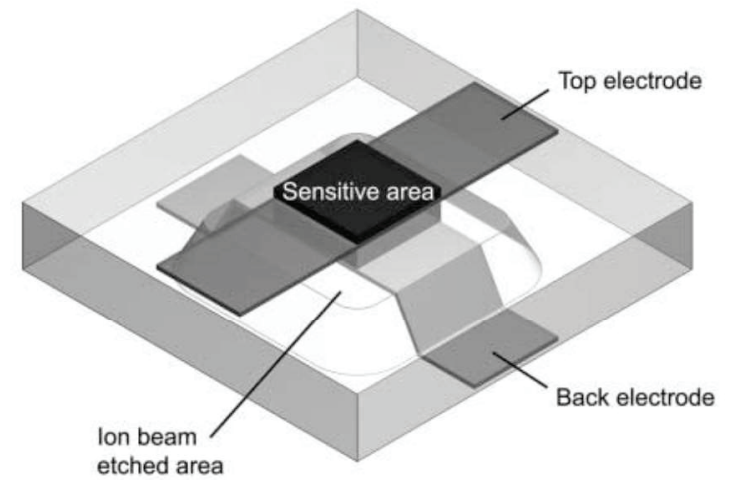

Figure 1: Sketch of the integration of the $L T$ sample (ion beam etched area) in a pyroelectric sensor.

\section{lon beam etching}

The 1.5" stainless steel precision subcarriers with the prepared LT wafers were mounted on a customized carrier which holds up to seven 1.5 " substrates to increase the throughput. The carrier with mounted substrates is shown in Figure 2. A thermal contact pad was used to couple the subcarriers efficiently to the main carrier. The remaining open area of the carrier is protected by a graphite plate which has a long life-time due to a low sputter rate. The ground plate is made of aluminum to keep it lightweight.

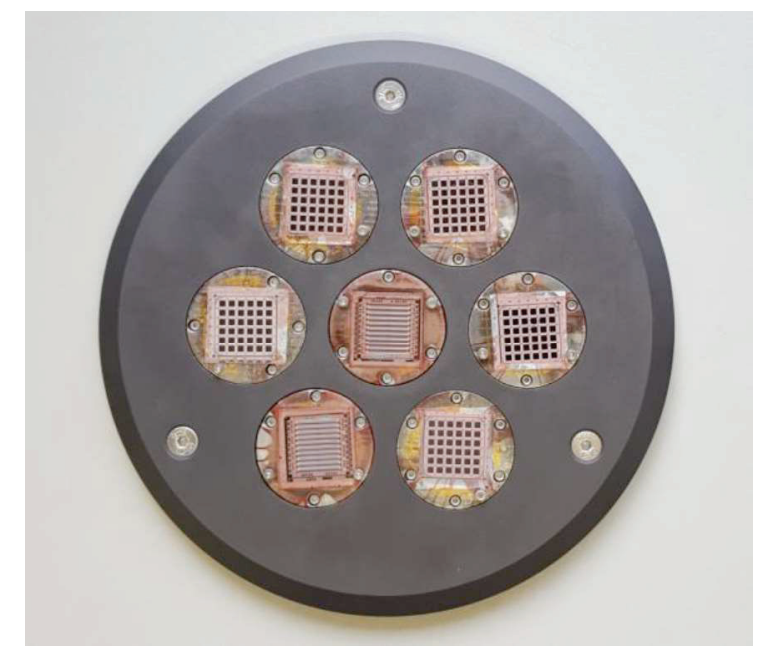

Figure 2: Carrier for seven sensor substrates prepared for ion beam etching.

The tool used for ion beam etching is a scia Mill 150 shown in Figure 3. It features automatic control of the tool by recipes and a high security standard, both required in production environment.

A size-optimized load lock is used for fast pumping to the handling pressure of $5 e-5$ mbar. At this pressure, the gate valve between the load-lock and process chamber is opened for handling the carrier.
A linear transfer system transports the carrier inside the chamber. A clamping mechanism fixes the carrier on the water-cooled substrate holder. Therefore, a venting of the process chamber for mounting the sample is not required. That prevents contaminations by particles and water. Besides, particle formation in the process chamber is minimized.

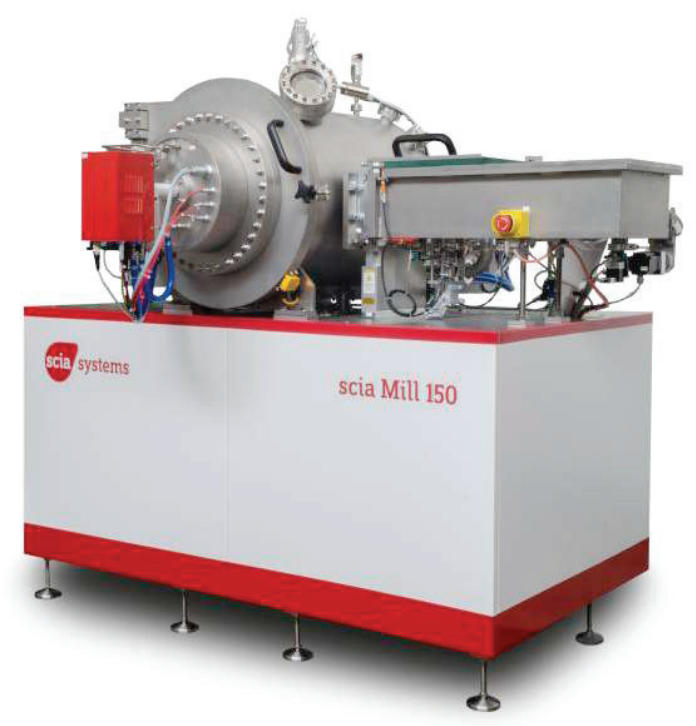

Figure 3: View of the scia Mill 150 used for the ion beam etching of the $L T$ substrates.

Due to the PR layer, the substrates have to be cooled efficiently during the ion beam etching. Since thermal conduction in vacuum is ineffective, helium is supplied between the carrier and the water-cooled substrate holder. Helium is (1) easy to handle and has (2) a high thermal conductivity. The helium buffer allows an efficient thermal coupling by convection.

We used a three-grid ion beam source (IBS) with a microwave excited argon plasma. The beam was extracted by applying a positive beam voltage on the innermost grid, called the screen grid which thereby defined the ion energy. The second grid, called accelerator grid, was used to shape the ion beam in order to get a minimum current on the second and the third grid. The third grid was grounded. The beam current ejected from the first grid minus the accelerator grid current is defined as the ion current. We used neutralizers supplied with argon for beam neutralization.

Before etching the sensors, a heating-up cycle of the source was applied over one hour. At this time, the IBS shutter was closed. The shutter was made of graphite and includes a current probe for ion current density measurements. 
The homogeneity of the ion beam source was determined by etching a 6" PR structured silicon wafer for twelve hours while the substrate rotated with $3 \mathrm{rpm}$. In Figure 4, the distribution of the removed material on the wafer is shown for an angle of incidence of $10^{\circ}$. The measurement was done by measuring two line scans using a profiler. A homogeneity of $0.7 \%$ (sigma/mean) across a 6 inch wafer was obtained with a mean removal rate of $1.3 \mu \mathrm{m} / \mathrm{h}$. Before these measurements, the optimization of the source was done by etching a thermally oxidized silicon wafers for three minutes after heating up the IBS. The obtained etching rate distribution and homogeneity was similar to the long-time process which confirms the stability of the IBS.

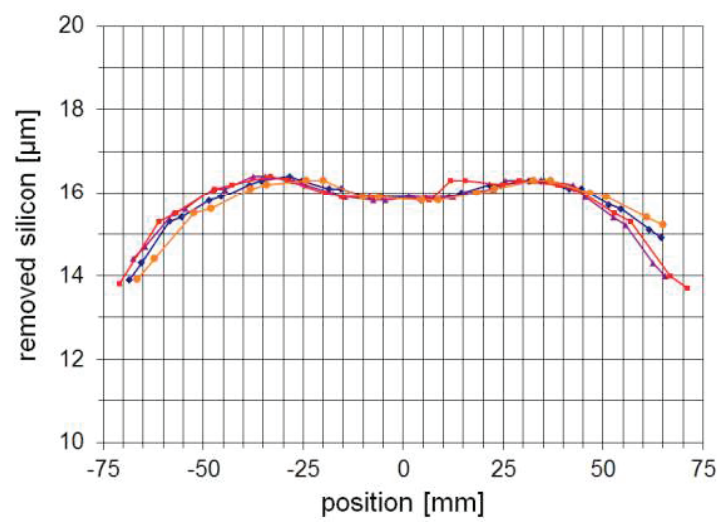

Figure 4: Removed silicon thickness after a 12 hour process.

\section{Results}

We etched about $15 \mu \mathrm{m}$ LT with a mean etching rate of $1.3 \mu \mathrm{m} / \mathrm{h}$. In the first step, a twelve hour etch was done. The microwave power regulated the ion current to a constant value of $265 \mathrm{~mA} / \mathrm{cm}^{2}$. In Figure 5, the power and ion current as a function of the etching time is shown. One can see a power drift over the first two hours which stabilized at about $360 \mathrm{~W}$. The mean ion current over 12 hours was $264.98 \mathrm{~mA}$ with a mean deviation from mean ion current of $0.37 \mathrm{~mA}$. This stability was required since the amount of removed LT was adjusted by the process time.

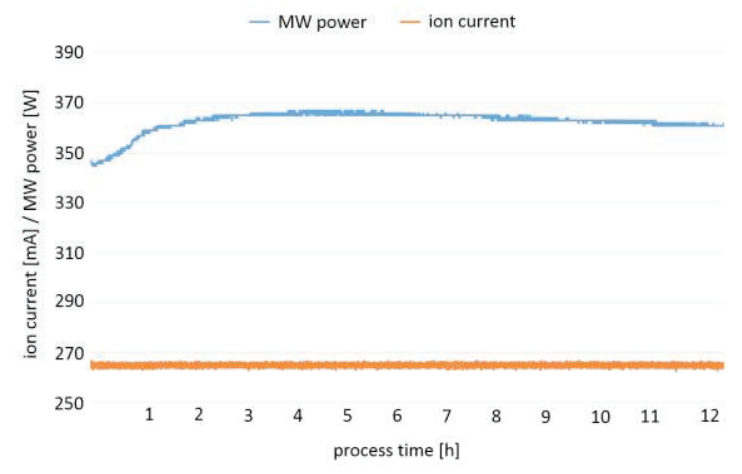

Figure 5: MW power as a function of process time for a regulated ion current of $265 \mathrm{~mA}$.
As the beam and accelerator current are an integral current of the grid, they hold no information about local changes of the ion current. To get this information, the shutter was closed every 15 minutes for one minute to measure the ion current in the center of the IBS. In Figure 6, the dependency of the ion current density on the process time for a twelve hour process is shown. Additionally, the deviation of the ion current density from its mean value was calculated. The same heating cycle and process parameters were used like for the process shown in Figure 5.

On can see that the measured ion current density varied about $+/-1.5 \%$ in twelve hours. The main shift happened in the first three hours after the heating up cycle and first shutter opening. While extracting the ion current density, the shutter had to be closed which influences the plasma properties inside the source. This effect caused a shift of the measured ion current density while the shutter was closed. This shift dominated the extracted ion current density in the first two hours. This is indicated by the arrow bars in Figure 6 .

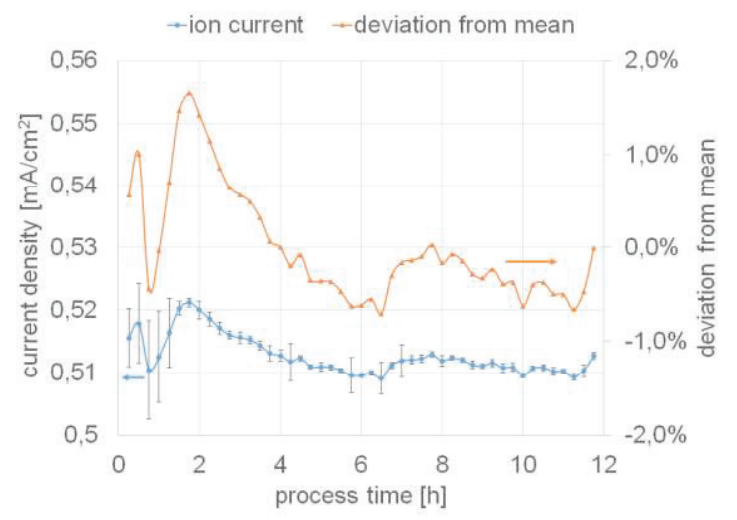

Figure 6: Dependency of the ion current density and its deviation from mean value for a twelve hour process.

After two hours, the error of the ion current density determination became smaller. At this time, a shift over one hour to an equilibriumlike ion current was observed. As the source was assumed to be in thermal equilibrium at this time, other effects like the observed power drift in Figure 5 and re-deposition of material on the third grid could lead to a small change of extracted current density in the center of the source.

\section{Analysis of the etched sensor elements}

After the ion beam etching, the substrates were analyzed in order to investigate the influence of the etching process. First the PR was visually examined. No PR was burned during the process. That indicates a good thermal coupling of the substrates to the substrate holder. 
The thickness of the LT was reduced from $25 \mu \mathrm{m}$ to $10 \mu \mathrm{m}$ determined by optical measurements. During the etching into the material, a wall developed whose shape could critically influence the electrical bonding later in the sensor production. In Figure 7 the smooth etching wall obtained at $15^{\circ}$ angle of incidence of the Argon ions was investigated by using a scanning electron microscopy. In the left image, a wall with sharp edges is observed. These sharp edges caused problems for electrically contacting the top electrode. Much better bonding results are obtained by using a modified PR mask which leads to a wall shape as shown in the right image.
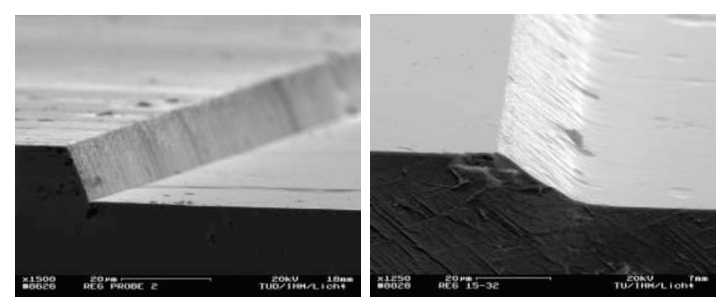

Figure 7: Scanning electron microscopy image of a $L T$ etch wall with (left) standard PR mask and (right) optimized PR mask. Both walls were obtained at $15^{\circ}$ angle of incidence.

By means of ion beam etching, the specific detectivity $D^{*}$ as an expression of the signal-tonoise ratio of a pyroelectric detector could be improved significantly. In Figure 8 it is shown, that an ion beam milled $5 \mu \mathrm{m}$ thin pyroelectric detector with a sensitive area of $2 \mathrm{~mm} \times 2 \mathrm{~mm}$ had a more than three times higher $D^{*}$ value than a non-milled $25 \mu \mathrm{m}$ thick detector. As a result, the ion beam etching technology opens new fields of application for pyroelectric detectors.

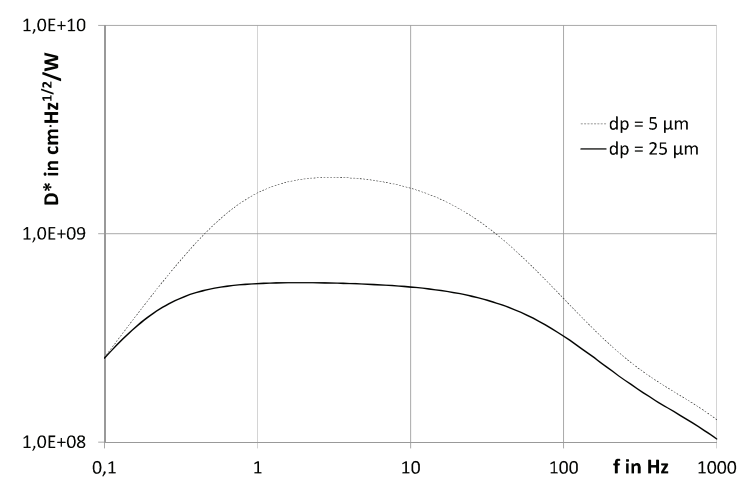

Figure 8: $D^{*}$ of $L T$ detectors with $2 \times 2 \mathrm{~mm}^{2}$ sensitive area and thickness $d_{p}$ of $5 \mu \mathrm{m}$ and $25 \mu \mathrm{m}$.

\section{Literature}

[1] Y. Kuwano et al., Japanese Journal of Applied Physics 20, 221-224 (1981)

[2] K. Fukuta et al., Japanese Journal of Applied Physics 20, 159-161 (1981)

[3] S. E. Stokowski et al., Infrared Physics 16, 331334 (1975), doi:10.1016/0020-0891(76)90056-7

[4] V. Norkus et al., tm-technische Messen 81, 99106 (2014), doi:10.1515/teme-2014-1018

[5] M. Schossig et al., tm-technische Messen 81, 127-136 (2014), doi:10.1515/teme-2014-1022 